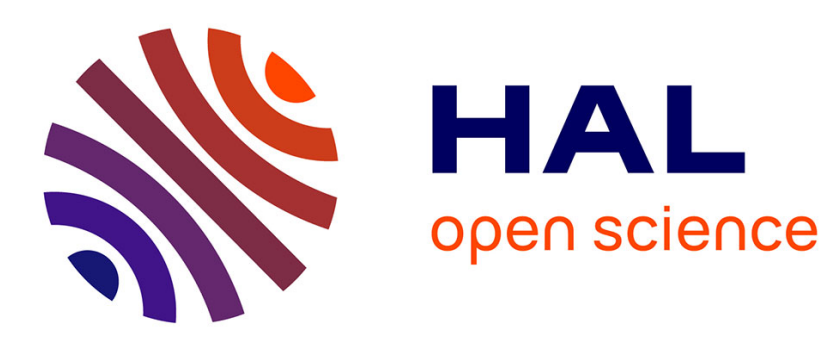

\title{
VÉRITÉ ET ILLUSION DES APPROCHES CROISÉES ENTRE PSYCHANALYSE ET NEUROSCIENCES
}

\author{
Entretien Avec, Roland Gori, Rémy Potier
}

\section{To cite this version:}

Entretien Avec, Roland Gori, Rémy Potier. VÉRITÉ ET ILLUSION DES APPROCHES CROISÉES ENTRE PSYCHANALYSE ET NEUROSCIENCES. Adolescence, 2011, 10.3917/ado.077.0531 . hal01484913

\section{HAL Id: hal-01484913 \\ https://hal.science/hal-01484913}

Submitted on 8 Mar 2017

HAL is a multi-disciplinary open access archive for the deposit and dissemination of scientific research documents, whether they are published or not. The documents may come from teaching and research institutions in France or abroad, or from public or private research centers.
L'archive ouverte pluridisciplinaire HAL, est destinée au dépôt et à la diffusion de documents scientifiques de niveau recherche, publiés ou non, émanant des établissements d'enseignement et de recherche français ou étrangers, des laboratoires publics ou privés. 


\section{VÉRITÉ ET ILLUSION DES APPROCHES CROISÉES ENTRE PSYCHANALYSE ET NEUROSCIENCES}

Entretien avec Roland Gori

Rémy Potier

Editions GREUPP | «Adolescence »

$2011 / 3 n^{\circ} 77$ | pages 531 à 544

ISSN 0751-7696

ISBN 9782847951998

Article disponible en ligne à l'adresse :

http://www.cairn.info/revue-adolescence-2011-3-page-531.htm

\section{Pour citer cet article :}

Rémy Potier, «Vérité et illusion des approches croisées entre psychanalyse et neurosciences. Entretien avec Roland Gori », Adolescence 2011/3 (n 77), p. 531-544. DOI 10.3917/ado.077.0531

Distribution électronique Cairn.info pour Editions GREUPP.

(C) Editions GREUPP. Tous droits réservés pour tous pays.

La reproduction ou représentation de cet article, notamment par photocopie, n'est autorisée que dans les limites des conditions générales d'utilisation du site ou, le cas échéant, des conditions générales de la licence souscrite par votre établissement. Toute autre reproduction ou représentation, en tout ou partie, sous quelque forme et de quelque manière que ce soit, est interdite sauf accord préalable et écrit de l'éditeur, en dehors des cas prévus par la législation en vigueur en France. Il est précisé que son stockage dans une base de données est également interdit. 


\section{VÉRITÉ ET ILLUSION DES APPROCHES CROISÉES ENTRE PSYCHANALYSE ET NEUROSCIENCES}

Entretien avec Roland Gori

RÉMY POTIER

- Rémy Potier : "Que penses-tu en général des approches croisées entre psychanalyse et neurosciences? Quel est selon toi l'heuristique de ce type de recherche?»

- Roland Gori : «Qu'est-ce qu'on entend par approches croisées? S'agit-il d'approches croisées ou d'approches complémentaires au sens épistémologique du terme ? Ce n'est pas pareil. S'il s'agit d'approches complémentaires, ça veut dire que chacun entreprend à partir de sa méthodologie de construire un objet, mais les superpositions sont dans ce cas aléatoires, liées à des similitudes de termes ou de thèmes idéologiques. Ça vient garantir de surcroît. Ou alors croisées au sens où les résultats des uns et des autres pourraient se valider réciproquement. C'est une question épistémologique difficile.

En général, je dirais qu'elles répondent aujourd'hui à un besoin social et à une nécessité culturelle. Il y a un souci de notre culture de trouver des ancrages matériels et si possible biologiques des processus psychiques et sociaux. Donc je pense que c'est davantage aujourd'hui un besoin idéologique qui se fait jour au travers de cette recherche. Je pense que ça se déduit beaucoup moins de la logique épistémologique de chacune de ces approches que d'une pression culturelle qui les amènerait à se confronter. La psychanalyse a été et est encore très présente dans la sphère sociale et politique voire médiatique, ce qui n'était pas d'abord le cas des neurosciences. Aujourd'hui, la psychanalyse subit un " choc" 
dans notre culture, qui est davantage un choc idéologique que la remise en cause de ses capacités thérapeutiques réelles ou de l'invalidité épistémologique réelle ou heuristique. Pour autant, la psychanalyse face à ce choc, recherche des alliés. Il y a presque un contrat idéologique et culturel qui est passé, de la part des neuroscientifiques, du type "Aideznous à populariser nos recherches et, en échange, on vous apportera le soutien dans la formalisation scientifique de votre démarche ". Il y aurait davantage un contrat idéologique qu'une nécessité épistémologique. »

- Rémy Potier : "Que penses-tu plus particulièrement de cet article de J. Dayan et B. Guillery-Girard, " Conduites adolescentes et développement cérébral : psychanalyse et neurosciences ", que publie la revue Adolescence? Il m'a semblé pour ma part que les auteurs étaient très soucieux des distinctions d'approches et de l'incommensurabilité de la démarche analytique avec d'autres disciplines?"

- Roland Gori : « Par rapport à cet article, d'une approche honnête, on pourrait se demander dans quelle mesure il répond à une nécessité des recherches - qu'on appelle croisées - ou bien s'il répond à une nécessité idéologique de rapprocher des champs dont l'un est en pointe culturelle, mais en même temps en déficit de confiance (la psychanalyse) et l'autre dans un crédit (assez illimité d'ailleurs) de confiance et sans trop d'influence médiatique.

Toute la question réside dans le fait de se demander ce que l'on entend par " croisé" : ça peut se croiser sans jamais se rejoindre ou bien effectivement, à un moment donné, se superposer. Croiser, ça ne veut pas dire grand-chose au-delà d'une croisade idéologique des deux champs. Pour la psychanalyse, l'enjeu serait de rattraper sa perte de vitesse scientifique et thérapeutique dans le champ idéologique. Pour les neurosciences, il s'agit finalement de s'appuyer sur les " primes de civilisation " qu'a pu avoir la psychanalyse, même si elle les perd actuellement. L'enjeu serait pour les neurosciences de gagner quelques parts de marché dans l'opinion publique. Le terme " croisé " montre bien qu'il est très énigmatique. Il s'agit plus d'une aventure idéologique que d'une nécessité épistémologique, surtout pour la psychanalyse. » 
- Rémy Potier : "Ce texte défend d'ailleurs une option développementale de la psychanalyse? Que peux-tu dire sur ce choix?" - Roland Gori : «Est-ce que ça vient renforcer la valeur du courant développementaliste pour la psychanalyse, ou est-ce que ça vient montrer qu'il y a des affinités électives entre des problématiques, ce qui n'est pas la même chose. Soit ça veut dire que le courant développemental en psychanalyse vient renforcer le courant naturaliste, soit ça veut dire que le courant développementaliste en psychanalyse entretient des affinités avec la psychologie ou avec les neurosciences. De la même manière que le structuralisme en psychanalyse entretient des affinités avec la philosophie ou avec la sociologie. Ce n'est donc pas pareil, d'un côté nous avons des affinités entre les théories et les méthodologies, de l'autre, nous avons des perspectives qui se renforcent dans leur solidité. Le sens n'est pas le même. Alliance idéologique des problématiques ou mise à l'épreuve de la validité scientifique?

L'approche génétique ou développementale est davantage soluble dans une psychologie psychanalytique à la Lagache que dans une théorie lacanienne. C'est un fait intéressant. »

- Rémy Potier : «Ton travail met l'accent sur les impacts sociaux et politiques du malaise contemporain, pourrais-tu dans ce cas précis expliciter l'enjeu de ces approches croisées? "

- Roland Gori : «On va chercher dans les sciences les facteurs de légitimation des choix politiques. Je ne suis pas convaincu que ce soit une bonne chose pour la démocratie. Les sciences peuvent apporter des éléments, pourquoi pas, mais je pense que, même si on en tient compte, il faut quand même s'en méfier. Je m'explique. Toutes les recherches, même celles qui concernent la psychanalyse, ne tiennent pas compte du contexte culturel et socio-politique des comportements. Par exemple, lorsqu'on nous dit qu'il y a une modification cérébrale majeure qui concerne le cortex préfrontal entre quinze et vingt-cinq ans, il faudrait ajouter, pour une culture donnée, à un moment donné, dans un contexte socio-politique donné. Or là, on crée la fiction d'un homme universel. Ceci étant, c'est la même chose avec la psychanalyse, c'est la même erreur d'approche : oubli du social et du culturel historiquement déterminés. 
C'est intéressant par ce que ça permet de débattre, mais en même temps je serais réticent d'aller dans le sens d'une validation réciproque par pure affinité des idéologies. Avec ce risque qui est de chercher la preuve par l'IRM et de gommer anthropologiquement la valeur de la parole.

Est-ce qu'on se situe au niveau des concepts qui peuvent se trouver remanier par les résultats d'autres sciences ou bien est-on dans l'illusion ?

À l'heure actuelle, on va avoir une floraison de ce type de tentative transdisciplinaire. Est-ce que ces approches sont dues à une conjoncture, à une stratégie de publication dans le contexte culturel actuel ou à un intérêt épistémologique ?

C'est la même question de fond qui se trouve pour moi posée : fairevaloir, mise en scène sociale ou nécessité impérieuse des méthodes? »

- Rémy Potier : "Serait-ce là l'une des illusions des approches croisées? De courir après la reconnaissance sociale?»

- Roland Gori : « La première illusion provient de l'utilisation de mots, de notions qui sont communes au plan sémantique, mais qui ne sont pas identiques du point de vue de leur définition conceptuelle. Comme le disait G. Bachelard, un même mot n'est pas un même concept, chaque fois il faut insérer les concepts dans un champ phénoméno-technique d'expériences.

Même si le texte est honnête dans sa présentation, la caractéristique de son approche du point de vue épistémologique est de réduire la polysémie. Or la caractéristique d'un concept, c'est de réduire sa polysémie en intégrant ce concept dans un champ de significations déterminé par la méthode et par sa solidarité aux autres concepts.

Lorsque les auteurs écrivent que " les études de neuro-imagerie ont fait apparaître des liens nouveaux entre puberté et adolescence. La puberté, souvent conçue uniquement comme un phénomène biologique de croissance et de sexuation du corps, a souvent été opposée à l'adolescence, phénomène psycho-social "1, tout dépend du sens que l'on

1. Cf. dans ce même numéro l'article de J. Dayan, B. Guillery-Girard (2011). Conduites adolescentes et développement cérébral : psychanalyse et neurosciences. Adolescence, 29 : 479-515 (p. 505). Les pages en regard des citations renvoient à cet article. 
donne à chacun de ces mots. Ou bien on est dans une sorte de soupe idéologique et alors on considère que l'adolescence existe à l'état d'évidence naturelle. Il y a une illusion réaliste : c'est-à-dire que des mots correspondraient à des références qui seraient limitées. Pas du tout. Tout dépend là aussi du sens donné aux mots « adolescence » et " puberté » : à dire que la cartographie de l'architecture cérébrale et de ses modifications confirme l'idée selon laquelle l'adolescence ne peut se réduire à une mutation sociologique, on fait passer des idées qui excèdent les données mêmes de l'expérience. C'est donc peut-être sur le caractère amphibologique des mots que l'on peut opérer un certain rapprochement, mais au risque de dégrader le concept en pure notion polysémique.

À un moment donné il est aussi question d'habitus à propos de la dépendance aux drogues ${ }^{2}$. Or l'usage de ce concept dans ce texte apparait assez distendu de la définition proposée par les sociologues. Chez N. Elias, l'interdépendance des individus joue aussi sur leur personnalité en leur imposant des réseaux préexistants, qui laisseront leur empreinte dans l'habitus de l'individu. Chez P. Bourdieu, c'est un système de dispositions socialement réglé, intériorisé. Donc le terme d'habitus, à partir de la sociologie et de l'anthropologie, est inséparable des normes, des conditions des civilisations et des mœurs. Problématique qui, contrastant avec la démarche actuelle et provisoire des neurosciences, désavoue l'Autre, le transubjectif. »

- Rémy Potier : "Sauf à mettre en avant le rôle de l'autre dans l'organisation cérébrale?"

- Roland Gori : " Oui, mais pour l'instant, les neurosciences ne semblent pas pouvoir s'en saisir, même si elles peuvent mettre le rôle de l'autre en avant : les neurones miroirs, l'épigénétique. Mais ce n'est jamais l'Autre, tel que nous l'entendons, ce n'est pas l'Autre du langage,

2. Par exemple : «Les comportements à risque peuvent être l'expression irrésistible d'un conflit inconscient, comme dans l'acting out, et sont alors la plupart du temps des événements isolés, ou résultent d'un habitus, qu'y soit ou non associée la notion de dépendance » (p. 486). 
ce n'est pas l'Autre de l'intersubjectivité, ni celui de la phénoménologie. On est dans une démarche très collée au modèle animal, au modèle médical du corps, c'est-à-dire le cadavre. L'approche par l'IRM est une approche du cadavre, même si c'est fonctionnel. On est toujours dans la même filiation : l'autre comme forme cadavérisée ou spéculaire.

Pour l'instant, en tout cas, on en est là. Je sais que certains au Collège de France, notamment, ont la prétention d'une neuroscience sociale, qui intègre la dimension sociale dans les recherches neuroscientifiques. Mais pour l'instant, il m'apparaît que c'est très distendu par rapport à ce que nous entendons par l'Autre et sousentendons par le signifiant, etc. »

- Rémy Potier : « Je comprends bien ton argument sur la polysémie conceptuelle. Il me semble que les auteurs expriment bien l'objet de leur tentative d'approche croisée dans la phrase suivante: "Si les neurosciences ne peuvent en aucune manière démontrer les hypothèses psychanalytiques, comme il est parfois affirmé, elles peuvent y porter un éclairage fécond qui prend sens du fait d'une 'métacognition commune', d'un savoir partagé sur l'homme " (p. 490). Peux-tu commenter et nous dire ce que tu penses en ce cas de l'idée d'une " métacognition commune " ?"

- Roland Gori : " Pourquoi chercher aujourd'hui à consolider des affinités qui paraissent plus idéologiques entre des domaines de connaissances, que résulter d'une nécessité épistémologique. Ça résume ma première réponse. Ça me parait très important, dans la mesure où ce type d'article vient faire une sélection à l'intérieur des théories psychanalytiques elles-mêmes en favorisant l'approche développementale par rapport à l'approche structurale. Là encore se pose la question de savoir si l'approche développementale est beaucoup plus juste que l'approche structurale. Estce la raison pour laquelle cette compatibilité existe entre les données des neurosciences et les données d'un point de vue génétique en psychanalyse ou bien est-ce partisan ? Parce qu'ils ont des affinités électives idéologiques? Il y aurait donc là l'illusion d'un champ commun avec pour conséquence de favoriser à l'intérieur d'un champ des théories ou des méthodes qui sont valorisées par un autre champ ! Je ne suis pas sûr qu'on y gagne en scientificité. » 
- Rémy Potier : "Peux-tu préciser la question de l'idéologie? 》

- Roland Gori : « Il y a aujourd'hui dans notre société un retour du positivisme et du scientisme tel qu'on a pu le connaître dans la deuxième moitié du XIX $\mathrm{X}^{\mathrm{e}}$ siècle. Le politique face à l'effacement de son autorité - déduite des grands états transcendantaux - cherche finalement dans les sciences la justification des décisions qu'il prend. On fait comme si le politique ne prenait pas partie, comme s'il ne faisait que gérer au mieux les intérêts de l'humain, or il justifie les décisions qu'il prend en s'appuyant sur la science. Ça me paraît extrêmement dangereux. Il s'agit d'une folie, de la " religion de la science" dont parlait déjà E. Renan. Il s'agit d'un des plus grands dangers pour la démocratie aujourd'hui. Une démocratie fondée sur la norme et non une démocratie fondée sur le politique de la parole.

Par conséquent, aller chercher, du côté des neurosciences, des éléments de base aux décisions juridiques, judiciaires, politiques, me paraît une monstruosité, du point de vue de la démocratie, entendue comme forme politique de gouvernement. Il y a donc aujourd'hui des programmes de neuro-loi. Citons l'exemple d'une femme en Inde qui a été condamnée sur la base des données d'IRM censées attester qu'elle mentait. Évoquons aussi le cas de deux sociétés, aux États-Unis, dont le slogan est " la vérité est notre business " et qui tentent de vendre aux tribunaux des preuves selon lesquelles les gens disent la vérité ou mentent. Mentir nécessite plus d'apport de glucose dans certaines zones cérébrales. On peut donc déterminer par IRM la probabilité que quelqu'un dise la vérité ou non. Les tribunaux ont pour l'instant refusé ce type de preuve. Il faut bien dire pour l'instant. Ces deux sociétés prospèrent et n'hésitent pas à vendre la " preuve par IRM " aux compagnies d'assurance ou aux compagnies qui l'utilisent pour des recrutements. »

- Rémy Potier : "Cet article me semble loin de ce type de projet, non, il a même une dimension politique intéressante, dans l'esprit de ce que tu défends par ailleurs et surtout à partir d'une tout autre épistémologie? Tu n'es pas d'accord?"

- Roland Gori : «Aller de ce côté, c'est-à-dire avancer à partir des données des neurosciences en disant qu'à l'adolescence, la prise de risque 
est concomitante de certains développements différentiels des zones cérébrales, ça va peut-être dans le sens de disculper l'adolescent de certaines de ses actions, mais je ne suis pas convaincu que ce soit une bonne chose de fonder cette tolérance sociale des peccadilles de l'adolescence, de la fonder sur les résultats des neurosciences comme de la psychanalyse. De la même façon qu'il est agaçant de voir des analystes conseiller le Prince pour telle ou telle décision politique. Chaque fois le pouvoir politique va faire son marché dans les savoirs et les pratiques en libre-service en choisissant finalement l'idéologie qui est la plus à même de servir le discours politique. Or la démocratie c'est une affaire de parole, c'est un consensus moral, social, culturel. Ce qui me frappe - sur ce point on peut trouver des points communs entre psychanalyse et neurosciences - c'est la construction d'un homme universel, complètement désinséré de son contexte social et de son contexte historique.

On a l'impression qu'en termes de cerveau comme en termes de psychanalyse, le concept d'histoire sociale n'existe pas. C'est très frappant. Il y a quelque chose d'extraordinaire dans l'usage du concept d'habitus dans ce texte, comme effet de la polysémie. Lorsque les auteurs écrivent que " dans le cas de l'habitus toxique, l'incertitude sur le plan social et la quête anxieuse de relations sexuelles et affectives tendent à disparaître pour être remplacées par des comportements conventionnels et ritualisés, aboutissant à une satisfaction obligatoire " (p. 486), nous nous trouvons selon moi dans des confusions conceptuelles extravagantes.

On le voit bien dans ce passage également, qui présente une vision purement biologique de l'adolescence, au détriment de son contexte social historique : "Les neurosciences mettent en évidence durant l'adolescence une réorganisation cérébrale majeure qualifiée de ' maturation'. Elle se situe électivement dans les zones du cerveau impliquées dans les tâches décisionnelles de haut niveau, qui sont aussi des aires associatives. Ce remaniement présente un double aspect. Le premier consiste principalement en la destruction massive de synapses (élagage synaptique). Elle débute pour le cortex frontal vers onze/douze ans, et semble s'achever vers vingt-trois/vingt-cinq ans. Elle est plus tardive pour le cortex temporal, avec un début vers quatorze/seize ans et une maturation achevée durant la troisième ou quatrième décennie. Le second 
aspect, corrélatif du premier, réside en l'important accroissement de la connectivité anatomique et fonctionnelle entre les aires cérébrales, phénomène qui se prolonge bien au-delà de l'adolescence. Pour expliquer les comportements spécifiques de l'adolescence, une des interprétations proposées par les neurosciences met en avant un déficit du contrôle cognitif. L'immaturité des structures corticales impliquées dans les processus décisionnels de haut niveau, situées principalement au sein du cortex préfrontal (CPF), les placerait sous l'influence excessive du système limbique, ainsi que des systèmes de récompense et de punition " (pp. 479-480).

Ce qui veut dire, en d'autres termes, qu'à l'intérieur des civilisations où l'âge moyen de l'expérience de vie était de trente ou quarante ans, l'on était dans ce cas adolescent toute sa vie. On voit bien que la tache aveugle des neurosciences et de certaines psychanalyses, à partir du moment où se construit un " homme universel", consiste à faire l'impasse totale sur l'histoire des civilisations, comme de l'espèce humaine, c'est-à-dire sur les différentes forces et rapports de force qui ont eu lieu à un moment donné dans une société donnée. Il y a des gens qui seraient morts adolescents pendant un pan entier de l'histoire. Notre espérance de vie, comme disait R. Canguilhem, n'est pas une norme qui se déduit de la moyenne, mais qui se traduit dans la moyenne. On voit bien comment on raisonne ici dans un contexte d'espérance de vie égale à environ quatrevingts ans. On oublie qu'il n'y a pas si longtemps encore, on ne vivait pas plus que cinquante ans. »

- Rémy Potier : "N’y aurait-il pas certaines épistémologies en neurosciences qui prendraient en compte la place tenue par l'environnement dans l'organisation cérébrale? Du fait de l'interaction de l'homme avec son environnement, on pourrait concevoir une organisation cérébrale qui soit pensée en contextualisant et en prenant en compte les données sociales historiques? Penser le cerveau en relation avec le contexte social historique, ça ne me semble pas une mauvaise idée. Dans le cas de l'imagerie médicale, on voit bien que les rapports médicaux ne prennent pas en compte la singularité et que les termes sont souvent irrecevables pour certains patients qui ne comprennent pas l'image. Je pense à un patient de quatre-vingts ans qui va passer une IRM 
et qui constate que son rapport fait mention d'une dégénérescence quelconque et tout à fait négligeable. Pourtant cette nouvelle le plonge dans une profonde dépression. Il y a un intérêt clinique majeur à penser les effets de normativité à partir de ces images qui sont censées dire comment est un cerveau. Qu'en penses-tu?»

- Roland Gori : « Ce sera peut-être l'avenir des neurosciences, mais dans cet article que nous commentons, la conception de l'adolescence est problématique. Il y a une réelle confusion entre le concept et le fait d'expérience. C'est tout le problème des sciences, qui ne pensent pas, mais calculent. Le problème c'est quand le scientifique parle. C'est le problème de la communication scientifique. Il y a une résurgence de la rhétorique au sein des discours scientifiques. Leur discours n'est pas totalement scientifique. Le discours qui s'appuie sur les données de la science interprète le monde. Et pourtant, on constate dans ce texte une vraie prudence et le souci de mettre en avant les limites de résultats partiels. Néanmoins, prenons l'exemple de cette phrase : " La découverte des caractéristiques anatomiques et fonctionnelles du remaniement cérébral spécifique de la période de l'adolescence offre un intérêt particulier à la mise en perspective des neurosciences et de la psychanalyse ". Cela demande à s'interroger sur ce que veut dire "intérêt particulier" et ce que signifie " période de l'adolescence". Si ça ne se situe pas au même niveau, qu'est-ce que veut dire cette tentative de mise en perspective des neurosciences et de la psychanalyse ?

Il faut interroger chacun de ces termes. Si on lit attentivement le texte en faisant attention aux dimensions épistémologiques et politiques, on voit bien les problèmes que cela pose. S'agit-il d'une approche croisée qui permet aux disciplines de se rejoindre, ou bien ce sont des travaux et des méthodes qui sont complémentaires, au sens de N. Bohr - au sens du principe de complémentarité - c'est-à-dire que les théories sont vraies, mais exclusives l'une de l'autre. C'est uniquement du point de vue de celui qui cherche des alliances idéologiques que s'opère l'illusion qu'elles pourraient un jour ou l'autre se rejoindre. C'est une discussion sur laquelle je ne souhaite pas trancher. Mais il me semble que c'est très important, d'autant plus dans le champ de la psychopathologie. Dans notre champ, on voit bien comment il y a sans cesse un mélange de critères hétérogènes. 
Le problème c'est que les enjeux deviennent très vite moraux plus que médicaux. C'est vrai que les auteurs en disent quelque chose au niveau de la troisième partie où ils abordent le comportement en montrant qu'il s'agit d'un objet indirect pour la psychanalyse. Il est très honnête d'écrire que “Quoi qu'il en soit, l'épistémologie scientifique contemporaine nous apprend qu'il n'existe pas de fait en soi, mais seulement des données d'observation construites avec l'observateur et recueillies dans un dispositif expérimental spécifique ". L'hétérogène est tel que même à l'intérieur d'un champ, l'objectivation prétendue est en effet des plus problématiques. Ce n'est pas en ajoutant d'autres champs qu'on va nettoyer l'hétérogénéité de ces critères. C'est le problème de cet article selon moi, qui, je le répète, ne présente aucune mauvaise intention mais comporte des risques de confusion par séduction réciproque.

C'est la critique majeure que j'adresserai à ces recherches, qui sont pourtant à la fois importantes et légitimes. Pendant trop longtemps le milieu psychanalytique les a condamnées par des a priori plus idéologiques que scientifiques. Il faut bien, d'un point de vue tant épistémologique que politique, admettre qu'elles ont des points d'impacts majeurs sur la dimension sociale, culturelle, anthropologique, historique, intersubjective. Mais je crois que ce n'est pas en faisant une espèce de " salade niçoise " qu'on arrivera à clarifier les choses. Dans ce texte, ce qui me parait illustrer cette tendance par exemple c'est la partie "Épidémiologie des comportements à risque". Selon moi, cette approche témoigne du fait que l'on est dans une culture très adultocentrée et au sein de laquelle le modèle pour penser la souffrance psychique demeure médical et de santé publique. »

- Rémy Potier : "Comment conjugues-tu l'aspect intéressant que tu reconnais et la dénonciation du fait que c'est une démarche qui rentre de plain-pied dans l'idéologie du moment? Comment éclairer l'ambiguïté de l'intérêt et du risque idéologiques dans ton esprit? N'est-ce pas là l'un des aspects de l'illusion de provoquer intérêt et risque à la fois?»

- Roland Gori : « Dire que c'est dans l'air du temps, que c'est du côté de l'idéologie que ça se passe, c'est une façon de se déprendre de 
l'illusion de ce que l'on pourrait attendre de la science. Ma position est une façon de refuser l'objectivation et la réification à laquelle notre société de consommation et de spectacle nous pousse.

Les approches croisées se situent au cœur des conceptions cognitives, qui pourtant ne devraient pas confisquer le terme de cognition comme c'est le cas aujourd'hui. La cognition, ça veut dire "je connais". La cognition ne doit pas être confisquée par les sciences cognitives. Les sciences cognitives sont un signifiant porteur qui rassemble de façon rapsodique des choses qui n'ont pas grand-chose à voir, que ce soit la linguistique, les sciences de l'information et de la communication, etc. Ça a produit la " corporéification " d'une illusion. Il y a désormais une exigence épistémologique qui est tout autant une éthique qu'une politique. Une politique de la discipline, mais aussi une politique de la cité. On ne parle pas de la même chose, mais c'est très intéressant qu'on se parle. Peut-être que cela produira, chez les uns et chez les autres, de nouvelles idées qui pourront trouver l'humus à partir duquel ces idées deviendront des concepts.

Il n'y a aucun rapport entre les concepts de la physique quantique par exemple et ceux de la psychanalyse. Ça n'a, sur le plan des concepts, strictement rien à voir. Par contre, c'est très intéressant sur le plan des principes : il n'est pas possible d'exclure une théories au nom d'une autre, sauf à reconnaitre qu'elles sont vraies l'une et l'autre, mais exclusives l'une de l'autre. Tout dépend donc de la façon dont on pose la question dans le dispositif et les conditions de formulation de la réponse. La chimie crée bien son objet, l'objet scientifique est un artéfact produit du dispositif qui le fabrique. À partir de ce moment-là, il ne faut pas se faire d'illusion. Le concept, l'objet, la démarche, on ne les trouve pas au niveau naturel. Donc on ne doit pas exporter ces concepts dans d'autres champs, c'est ma position. Mais on peut débattre pour clarifier ses propres concepts.

Évidemment, à propos de ce que les auteurs appellent " métacognition ", je ne suis pas en accord. Comme " pousse-à-penser", oui bien sûr. Je dirais plutôt " discussion ", c'est-à-dire lieu citoyen. Selon moi, chaque démarche doit apparaitre dans son caractère spécifique et local. La psychanalyse en premier lieu. Ce qui tue la psychanalyse, ce n'est pas simplement cette logique sécuritaire contemporaine dans le 
monde de la santé, c'est aussi l'arrogance des psychanalystes qui avaient une vision du monde qui faisait l'impasse sur tout le reste : le politique, le social, le biologique, etc. »

- Rémy Potier : "Peux-tu préciser ton propos, concernant les problèmes posés par ces illusions d'ordre épistémologique?»

- Roland Gori : « Il me semble qu'il y a au moins deux problèmes. Un problème interne à chacune des épistémologies - correspondance ou corrélation ? Ce n'est pas la même chose. Par exemple, mettre en correspondance des fonctionnements différentiels des zones cérébrales pour certains types de comportements, ce n'est pas la même chose que les mettre en corrélation, c'est-à-dire, à partir de variables expérimentales, permettre de déterminer une causalité ou une corrélation. Par exemple, il $\mathrm{y}$ a eu des hypothèses en débat concernant la schizophrénie à partir d'approches neuroscientifiques. Le comportement répétitif du schizophrène implique-t-il des zones spécifiques du cerveau ou au contraire, le sculpte, le marbre cérébral détermine-t-il la structure du comportement ? Il y a bien un débat épistémologique au niveau des neurosciences qui est dans cette logique très intéressant.

On retrouve le même débat dans la psychanalyse, en 1920, avec le cas de la jeune homosexuelle ${ }^{3}$. Freud avance l'idée qu'on peut dire dans une analyse après-coup et non à l'avance, que le fait de voir sa mère tomber enceinte, provoquera chez sa fille une identification au père par dépit. Freud dit bien que c'est rétrospectivement qu'on peut construire des hypothèses pour dire ce qui s'est passé pour quelqu'un singulièrement. Pour autant on ne peut pas en tirer le principe selon lequel, quand ça se produit d'une telle façon dans l'histoire, ça produit tel type de problème psychique ou autre.

À l'intérieur même de chaque discipline se posent des problèmes épineux, comme celui de la causalité. Si à l'intérieur de chaque discipline, il est difficile de dire ce qui résulterait de corrélation, de correspondance ou de

3. Freud S. (1920). Sur la psychogenèse d'un cas d'homosexualité féminine. In : Névrose, psychose et perversion. Paris : PUF, 1997, pp. 245-270. 
coïncidence, alors qu'en est-il a fortiori pour passer d'une discipline à l'autre? Il est indispensable que les chercheurs se parlent, se rencontrent, mais sans rien céder à la rigueur épistémologique spécifique de leurs démarches.

Pour conclure par l'exemple et dire ce que je considère comme un bon usage des approches croisées, je peux renvoyer à l'initiative autour du concept de consentement. On a interrogé le concept selon plusieurs logiques épistémologiques sans tenter de produire une synthèse artificielle, chaque auteur déployant sa conception du mot, en vertu de sa pratique et de son épistémologie. Ça me semble beaucoup plus fécond. »

- Rémy Potier : « Roland, je te remercie pour cet entretien. »

Rémy Potier

Univ. Paris Diderot, Sorbonne Paris Cité

Centre de Recherches Psychanalyse et Médecine

75010 Paris, France

potier.r@free.fr 